\title{
Grating mirror for diffraction of electrons
}

\author{
M. A. R. Krielaart* and P. Kruit \\ Faculty of Applied Sciences, Delft University of Technology, Lorentzweg 1, 2628 CJ, Delft, The Netherlands
}

(Received 9 August 2018; revised manuscript received 5 October 2018; published 5 December 2018)

\begin{abstract}
The ability to imprint a phase pattern onto a coherent electron wave would find many applications in electron optics, in analogy to what is already possible with photons in light optics. Spatially dependent phase manipulation is achieved in transmission electron microscopy by passing the beam through a phase plate. However, in transmission mode this technique suffers from crystal imperfections and electron-matter interaction. If instead the electron wave is reflected of a spatially modulated potential, these difficulties can be circumvented. To demonstrate this principle, we consider here a periodic topological mirror structure that results in a sinusoidal plane of reflection for the incident electron. The reflection of the electron then takes place just above the physical mirror surface. Such "electron grating mirror" is expected to diffract the incident wave upon reflection by the introduced path length difference. The mirror can then be used as an electron beam splitter and coupler, analogous to semitransparent mirrors used in light optics. This enables for instance a lossless Mach-Zehnder interferometer for electrons. A numerical model that solves the Schrödinger equation for this system is obtained to enable a quantitative description of the grating mirror. The results show that the obtained diffraction order intensities behave like squared Bessel function of their respective order, and thus for instance the results show how an increase in grating pitch reduces the sensitivity to energy spread in the incident electron beam. Additionally, we show how the use of the WKB approximation enables faster calculations in the case of general patterns.
\end{abstract}

DOI: 10.1103/PhysRevA.98.063806

\section{INTRODUCTION}

Quantum electron microscopy (QEM) aims at the interaction free imaging of beam sensitive materials [1]. To realize this technique, new electron optical devices are required. One of these new devices is a grating mirror for electrons. The physics of an electron grating mirror is not described before and we will introduce it here. We show how a grating mirror can be used to reflect an incident coherent electron beam and subsequently rearrange the amplitude of the several created sub-beams.

The grating mirror consists of a topological pattern of rectangular bars that is kept at a potential just above the beam energy, thus effectively creating a periodically modulated mirror potential in space. Alternatively, a grating mirror can be realized by a pattern of lines kept at varying voltage levels but this is not treated here. In QEM, a grating mirror fulfills the role of coherent beam splitter and coupler: it enables the transfer of the beam intensity from a reference beam into a sample beam and vice versa, analogous to an optical beam splitter found in interference experiments.

\section{A. Grating parameters}

The geometry of the grating mirror [Figs. 1(a) and 1(b)] is described by a pattern pitch $(p)$ and amplitude $(\delta)$. To

\footnotetext{
*m.a.r.krielaart@tudelft.nl

Published by the American Physical Society under the terms of the Creative Commons Attribution 4.0 International license. Further distribution of this work must maintain attribution to the author(s) and the published article's title, journal citation, and DOI.
}

obtain an order of magnitude estimate for these parameters, we draw an analogy with a multiple slit experiment. Here, the angles of the $k$ vectors with the optical axis are related via the pitch and wavelength $(\lambda)$ by approximately $\theta \approx \lambda / p$. The resulting diffraction pattern can than be imaged onto a screen via a (system of) lens(es). The diffraction spot separation is then approximately given by $d_{\text {spot }} \approx \theta f$, where $f$ is the focal length of the lens system.

For initial experiments, we will use low beam energies (1-4 keV) and MEMS fabricated lenses. Plugging in these energies and the small $(10-30 \mathrm{~mm})$ focal lengths of the lenses, typical values for pitch are in the range of $p=100-500$ $\mathrm{nm}$. This should then result in spot separations on our detector screen in the order of micrometers. When the pattern amplitude $\delta$ is then in the same order of magnitude as the pitch, the resulting equipotentials that form above the physical mirror structure exhibit the required sinusoidal shape. Grating mirrors with pitch and amplitude in the range mentioned can be fabricated from a flat silicon waver using lithography processes. An electrostatic potential is applied to the pattern and the resulting field is confined in space by a (grounded) field-limiting aperture, placed at a distance $d$ above the pattern.

For $\{p, \delta\} \ll d$, the electric field in the confined region can be approximated analytical by solving the Laplace equation. To do so, we describe the potential applied to the pattern on a straight line by

$$
U(0, y)=U_{p}\left[1+\frac{\delta}{d} \cos \left(\frac{2 \pi}{\text { pitch }} y\right)\right] .
$$

Here, $U_{p}$ is the pattern bias potential (a constant) and the second term modulates the potential to mimic the presence 
(a)

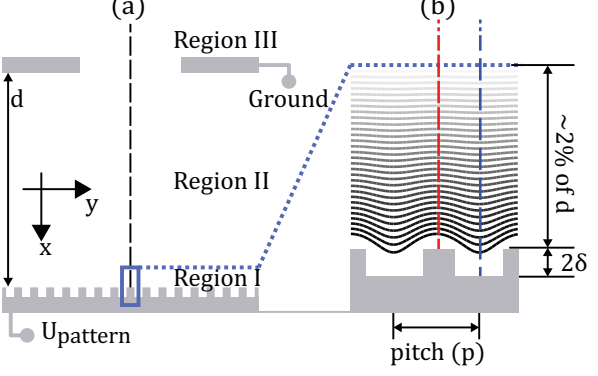

(c)

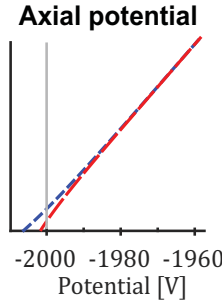

FIG. 1. (a) Parameters describing the grating mirror and coordinate system. (b) Details of region I, defining the pitch and amplitude of the pattern. Equipotential lines are shown schematically. (c) Axial and side potential corresponding to the details of region I.

of the pattern. By using separation of variables, the potential inside the confined region can then be shown to satisfy

$$
U(x, y)=U_{p}\left\{1+d^{-1}\left[\delta \sin \left(k_{p} y\right) \exp \left(k_{p} x\right)-x\right]\right\} .
$$

This result is valid for $x<0, \forall y$. The wave number $k_{p}$ is directly related to the pitch of the pattern, $k_{p}=2 \pi /$ pitch. From Eq. (2) it is observed that the modulation of the potential attenuates exponentially fast away from the mirror surface (region I in Fig. 1). For a distance $5 / k_{p} \ll d$ above the pattern (region II) the equipotential surfaces are virtually flat again.

An electron incident to the mirror decelerates first on the linear potential ramp inside region II. By spreading out the electron beam over the pattern, path length differences occur within the electron wave as it is being reflected inside region I. It is expected that this leads to a modulation of the phase of the wave function of the electron with corresponding diffraction effects in the far field. As the mirror is an amplitude splitter, consecutive illumination is expected to result in a coherent buildup of phase modulation, leading to increased probability amplitudes in the diffracted beams.

In this paper, we describe quantitatively how the pattern parameters (pitch, amplitude, applied potential, and field strength) dictate the physics of the electron grating mirror.

\section{METHODS}

Approximation methods, such as WKB [2] or convolution methods [3], allow for first-order estimation of the pattern effects. When applied along one spatial dimension parallel to the optical axis of the system [see Fig. 2(a)], the WKB method yields an integral over the selected electron trajectory that calculates the accumulated phase of an electron with given momentum $p(x)=\sqrt{2 m_{e} K}$ and $K$ the kinetic energy of the electron, related by

$$
\phi(x, y)=\hbar^{-1} \int p(x, y) d x .
$$

Here, the transverse coordinate $y$ is treated as a parameter that allows one to select different paths parallel to the optical axis. The phase modulation in the presence of the grating mirror can now be calculated by integration along various parallel paths starting and ending at the interface of regions I and II. This reveals an increase in phase modulation as (a)

(b)

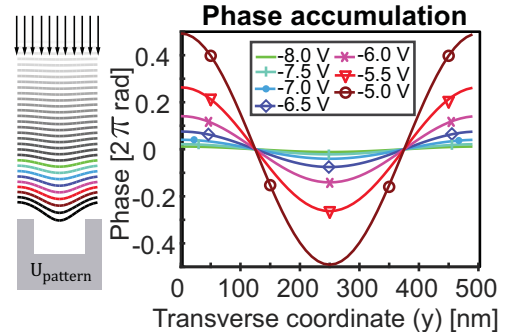

(c)

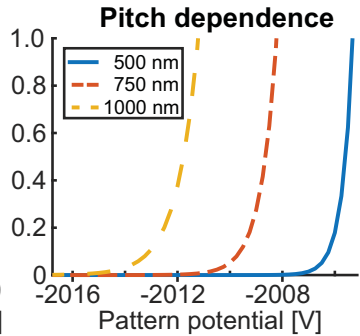

FIG. 2. (a) WKB approximation with integral paths parallel to the optical axis of the system. The arrows indicate (schematically) the starting point for the integration. (b) Obtained phase accumulation as a function of pattern potential (legend items with respect to $-2000 \mathrm{~V}$ beam energy) and transverse coordinate. The offset is subtracted such that only the modulated part of the phase is shown. (c) The effect of grating pitch on the relative phase accumulation.

the spread out electron reflects closer to the physical grating structure [Figs. 2(b) and 2(c)] as may physically be expected.

When the incident electron is described as a plane wave, $\psi_{\text {in }}=a \exp \left(i k_{\text {in }} x_{0}\right)$, where $k_{\text {in }}$ is the appropriate wave number, then the obtained spatial phase modulation $\phi(x, y)$ from Eq. (3) enables us to write the reflected wave at the interface of regions I and II $\left(x_{0}\right)$ as $\psi_{\text {out }}=a \exp \left[i \phi\left(x_{0}, y\right)\right]$. A Fourier transform of the reflected wave with imprinted phase pattern $\phi(x, y)$ then yields the spectrum of all $k$ vectors and their intensities.

However, the WKB approximation is generally not valid in the vicinity of the classical turning point of the electron just in front of the pattern [2]. This follows from the WKB ansatz, resulting in an inverse proportionality of the amplitude of the wave function and momentum $p$. Since the momentum $p(x, y) \rightarrow 0$ at the turning point, the wave function diverges here. It is just at this location, where the influence of the mirror pattern is dominant. Because of this, we do not trust a priori results obtained by using the WKB method. For this reason we also calculate the effect of the mirror potential by numerically solving the Schrödinger equation inside region I by coupling it to known analytical solutions of region II. Details of this more time-consuming calculation are included in Appendix A. Afterward, we compare the results of the WKB and numerical method in order to validate the use of the WKB method.

\section{RESULTS}

In the full numerical solution of the Schrödinger equation, we describe the interface between region I and region II of respectively unknown and known solutions by the wave function $\psi_{b}\left(x_{b}, y\right)$ as

$$
\begin{aligned}
\psi_{b}\left(x_{b}, y\right)= & \sum_{n} A_{n} \operatorname{Ai}^{-}\left(u_{n}\left(x_{b}\right)\right) \exp \left(i k_{y, n} y\right) \\
& +\sum_{n} B_{n} \mathrm{Ai}^{+}\left(u_{n}\left(x_{b}\right)\right) \exp \left(i k_{y, n} y\right) .
\end{aligned}
$$

Here, the coefficients $A_{n}$ represent probability amplitudes of incident waves with wave vector $\vec{k}$ determined by the angle with respect to the optical axis. Coefficients $B_{n}$ are obtained 

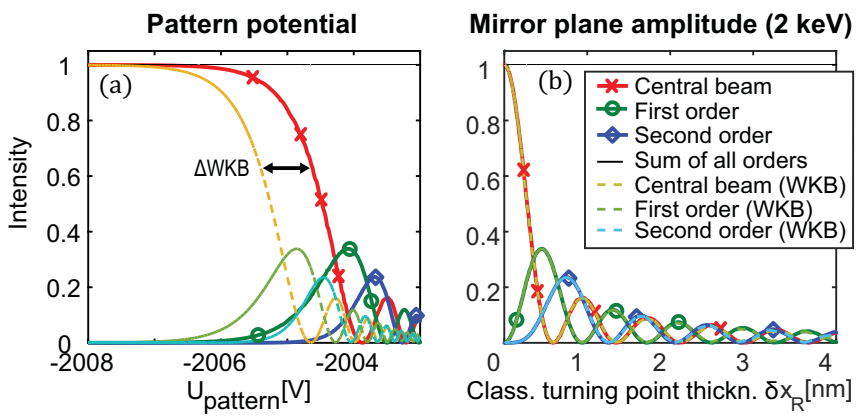

FIG. 3. (a) Intensity of the four most dominant orders as function of pattern potential. Solid lines (direct solution) and dashed lines (WKB approximation) of the Schrödinger equation are shown. (b) The amplitude $\left(\delta x_{R}\right)$ of the classical mirror plane $U_{\text {class. }}=\mathcal{E} / e$ $[\mathrm{V}]$ and corresponding diffraction order intensities are shown.

from the calculation and yield directly the probability amplitudes of the respective $k$ vectors of reflected waves.

When we consider an incident plane wave that travels parallel to the optical axis, all coefficients $A_{n}=0$, except for $A_{0}=1$. In the following, a field free electron energy of $\mathcal{E}=2 \mathrm{keV}$ is assumed. This requires a pattern potential of $U_{p} \leqslant-2 \mathrm{kV}$. When we assume a field strength of $10 \mathrm{kV} / \mathrm{mm}$, the field limiting aperture is positioned at a distance $d=200 \mu \mathrm{m}$ above the grating mirror.

The effect of a $500 \mathrm{~nm}$ pitch grating pattern is studied for various pattern potentials in order to show the effect of field modulation on the reflected electron. The intensities $I_{n}=\operatorname{conj}\left(B_{n}\right) \cdot B_{n}$ of the most dominant diffraction orders $(n=0, \pm 1, \pm 2)$ are plotted as a function of the pattern potential [Fig. 3(a)]. From this, it is observed that a maximum intensity of $34 \%$ is obtained in the first-order diffracted beam. This compares to transmission studies of diffractive gratings [4] and motivates the term "electron grating mirror" for the studied device.

To study the effect of a tilt angle between the incident beam and the normal of the mirror surface, two options are considered. First, we set all but one of the $A_{n}$ coefficients to zero. This simulates the effect entering the mirror field at exactly one allowed angle. The results of this simulation (not shown) indicate no sensitivity to angle of incidence but rather shift the entire spectrum of coefficients $B_{n}$ such that it is centered around the selected incidence coefficient $A_{n}$. Next, the effect of a tilt angle between the incident beam and the normal of the mirror that is smaller than the first-order angle is studied. This is possible by adding multiple pitches to the numerical domain. Again, it is found that the resulting spectrum of $B_{n}$ is centered around the incidence coefficient.

The diffractive properties of the mirror invite one to treat the system in a way similar to a diffractive crystal. One then studies the intensity of the various orders of diffraction as functions of the crystal thickness. Here, we consider the amplitude of classical mirror plane equipotential (given by $U_{\text {class. }}=\mathcal{E} / e$ ) as effective "crystal" thickness. The thickness $\delta x_{R}$ of the crystal can then be tuned by changing the pattern potential.

In Fig. 3(b) the diffraction intensities are plotted as a function of this effective thickness $\delta x_{R}$. As it can be seen from the

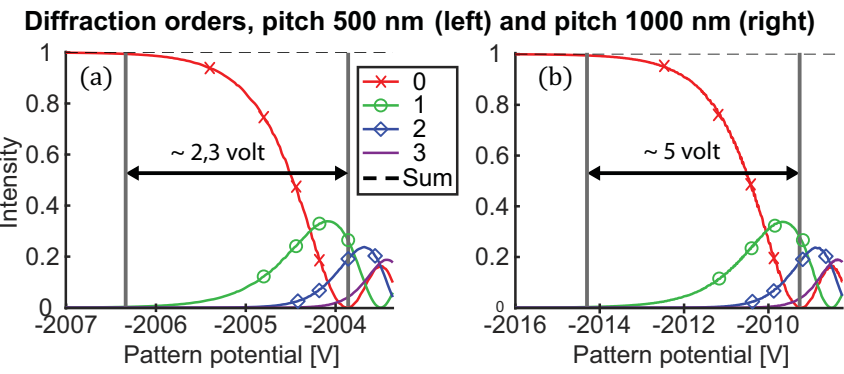

FIG. 4. Diffraction order intensities for a fixed pitch of (a) $500 \mathrm{~nm}$ and (b) $1000 \mathrm{~nm}$. An increase in pitch results in a wider bias potential window for complete attenuation of the central order beam.

figure, the intensity in the first-order diffracted beam increases linearly as a function of effective thickness for amplitudes close to zero. This linear increase is at the expense of intensity of the zeroth-order (central) beam and can be interpreted as an exchange of intensity between the two modes. In this range of effective thickness, the grating mirror can be applied as a nonsymmetrical beam splitter, meaning that a non-50:50 splitting ratio is achieved. It should be noted though that both the positive and negative orders are generated equally.

Earlier, we derived the exponential attenuation of the field modulation as function of pitch. As a result, it should be expected that the pitch influences the bias potential interval, over which the central beam becomes fully attenuated. In Fig. 4 we show the intensity of the first orders of diffraction for a grating mirror with pitch of $500 \mathrm{~nm}$ and $1000 \mathrm{~nm}$. Indeed it is evident from the plots shown that an increase in pitch stretches the interval over which the first-order diffracted beam is generated, from approximately $2.3 \mathrm{~V}$ to $5.0 \mathrm{~V}$.

The implications of this last result for an experiment are evident when one considers the energy spread inherent to an electron beam. By increasing the pitch, the sensitivity for energy spread is reduced, leading to better spatially defined diffraction spots. However, by increasing the pitch, one also has to increase the width of the beam that is spread out over the mirror surface, in order to illuminate an equal number of pitches when compared to the smaller pitch. As an alternative to increasing the pitch, one can also increase the field strength between the mirror and field-limiting aperture, by reducing the distance between the two or applying a positive bias to the field limiting aperture.

The above results are all obtained from the numerical solution of the Schrödinger equation, which is a computationally demanding procedure. We now compare the obtained results to the outcome of the WKB method in order to assert the validity of the latter. For this, we consider again a single pitch of the grating mirror. We use our analytical expression for the equipotential above the grating mirror [Eq. (2)] and integrate the momentum along paths running parallel to the optical axis, in order to obtain the spatial phase modulation. The obtained phase profile is then decomposed in the various spectral components using Fourier analysis and this yields the intensity of the diffracted beams.

In Fig. 3(a), the intensity of the central-, first-, and secondorder beams (dashed lines) are plotted as a function of 


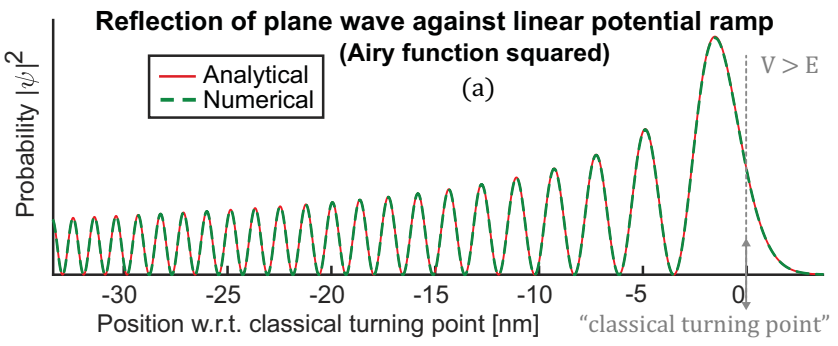

(b)

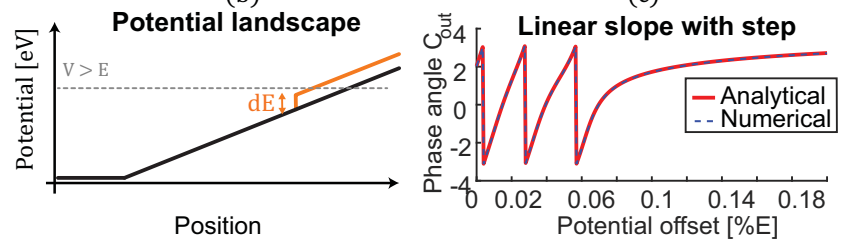

FIG. 5. (a) Airy function of first kind squared is plotted. The classical turning point coordinate is indicated. Notice that the reflected electron has finite possibility of reaching behind this point. (b) 1D potential ramp starting in free space. The gray line indicates the energy that corresponds to the classical turning point of the electron. (c) Comparison between analytical and numerically obtained phase angles for the potential bump at $x_{0}$, obtained at $x_{s}$.

applied pattern potential. Comparison to the exact solution (solid lines) shows good qualitative agreement. Quantitatively, however, the WKB result is shifted approximately $1 \mathrm{~V}$ with respect to the exact solution. It thus appears that the electron approaches the mirror more closely in the WKB approximation as compared to the exact case. Indeed, this is the case when one considers the Airy function solution of the exact case. The maximum probability $\psi^{*} \psi$ of finding the electron is positioned not at, but just before the classical turning point [also see Fig. 5(a)] and thus the electron spends more time at this point than at the classical turning point itself.

From the above, it appears to be evident that the WKB approximation does not yield useful quantitative results when the beam intensities are expressed as a function of applied pattern potential. However, we found that by expressing the beam intensities as functions of classical turning point thickness $\left(\delta x_{R}\right)$ instead, a perfect agreement between the WKB and exact method is obtained, as shown in Fig. 3(b). One can then calculate the potential necessary for this thickness in hindsight.

\section{DISCUSSION}

The physics of a constant pitch grating mirror is studied via two different methods. We observe a physical correspondence of the studied grating mirror with that of a diffractive crystal. Different pattern parameters are studied as well and these show similar results.

It should be noted that the diffraction order intensities as functions of effective crystal thickness [Fig. 3(b)] are remarkably similar to the square of Bessel functions of corresponding orders. This may suggest that an analytical solution for the studied system exists. This would enable a general description of diffraction order intensities for any periodic grating mirror.
We demonstrated in the previous section that the $\mathrm{WKB}$ and exact method show remarkable quantitative agreement, when the beam intensities are expressed as a function of classical turning point thickness instead of applied bias potential. This observation allows speeding up of future calculations, since the calculation of the WKB integral takes only a few seconds. This is an improvement over the use of exact solutions, the calculation of which takes up to 30 min on the PC used for the shown calculations. Additionally, the WKB method allows more flexibility for studying nonperiodic patterns.

Finally, we discuss several effects which might prevent us from obtaining the coherent diffraction as we described. The most obvious disturbing effect is a possible low-frequency distortion of the mirror topology, for instance, the effect of curvature of the surface due to stress inside the material. We can approximate the maximum allowable curvature by assuming illumination of the mirror with a beam of $20 \mu \mathrm{m}$ diameter. Then, for the generation of the first-order diffracted beam, a field modulation of the classical turning point of $0.5 \mathrm{~nm}$ is required [5]. From Eq. (2), we can then estimate a maximum thickness variation of $1 \mathrm{~nm}$ is tolerated within this region. A further increase in curvature will ultimately lead to blurring of the diffracted beams in the image plane of the mirror system. Similarly, the potential on the surface of the mirror is assumed to be very uniform. At $10 \mathrm{kV} / \mathrm{mm}$, a topology change of $1 \mathrm{~nm}$, as just discussed, is equivalent to a potential change of $10 \mathrm{mV}$. This sets requirements for the surface treatment of the mirror surface. Also, contamination should be avoided because this could lead to local charging. In principle, the electron beam does not need to touch the mirror surface ever, but during the alignment procedures it will be hard to really avoid electrons reaching the surface.

A more subtle effect is that of the induced mirror charges inside the mirror. At the sub-nA current that will be used, electrons can be treated as arriving individually. The proximity of an electron near a wall induces an image charge (distribution) inside this wall [6]. At the point of nearest approach to the mirror surface (approximately $500 \mathrm{~nm}$ when only the firstorder diffracted beam is induced) this yields an increase of the potential at the classical turning point. A simple calculation shows that this is in the order of millivolts. The force from the mirror charge on the electron is always perpendicular to the surface, so we do not expect any effect from this. However, as the electron moves towards and away from the mirror, the induced surface charges on the mirror surface are dragged along, possibly causing dissipation [7,8]. In the which-way setup of [8], this dissipation due to Joule heating is recognized as the mechanism that leads to dephasing of the state of the wave function associated with the respective path. As a result, decoherence is observed as the electron propagates alongside the wall.

A similar effect is observed for intensity splitting in nanofabricated gratings [9]. Here, the dissipative effect of the image charge can be related to the side walls of the slits that the electron passes through. The question is if this effect could cause decoherence in our proposed setup. The effect would be that a localized excitation in the mirror surface would cause the electron to lose energy, in the process collapsing the wave function to a smaller area than the original $20 \mu \mathrm{m}$. This would affect the diffraction. We do not have a full theory from which 
we can derive the probability of this effect. However, we point out that, in all of the cases in the literature, the dephasing of the wave function is the result of splitting the wave function and successive dissipation in one of the resulting trajectories as it travels for some distance parallel and very close to a surface. As this is not the case for the reflective grating mirror that we present here, we expect that the probability of decoherence will be smaller than in those cases. Also, earlier experimental work involving electron holography [3] with reflected electrons did not indicate any effects of decoherence.

A final effect to be considered is bremsstrahlung, the emission of a photon while decelerating and accelerating the electron. Potentially this could cause energy spread or local collapse and thus loss of coherence. However, a nonrelativistic estimate of the average energy that is lost per electron to bremsstrahlung for an initial electron energy of $2 \mathrm{keV}$ reflected in a $10 \mathrm{kV} / \mathrm{mm}$ field gives $1 \times 10^{-9} \mathrm{eV}$, which can safely be neglected.

Further requirements on experimental setups for demonstration of the grating mirror for electrons would require stable power supplies, with an accuracy that is well below the energy spread of the electron beam. This criterion is easily satisfied with modern supplies and the optional use of an additional low-pass filter on the output.

This all said, we must admit that we have tried to observe the diffraction experimentally and so far have not obtained convincing results. Future work requires experimental verification of the grating mirror. This demands the microfabrication of the grating mirror and (lens) apertures. The spatial coherence of the electron beam should then extend across a distance of multiple pitches. From our simulations it is obtained that a small pitch requires the electron to approach the mirror to such proximity that higher-order diffracted beams may not be generated. On the other hand, a too large pitch places a more demanding condition on spatial beam coherence.

The realization of a quantum electron microscope can benefit from the presented work, as we describe accurately the intensity of the diffracted beams for given operation parameters. One should however take into account the coherent buildup of signal in the higher-order diffracted beams [10].

\section{ACKNOWLEDGMENTS}

M.A.R.K. would like to thank J. Thijssen of the Theoretical Physics group at TU Delft for helpful discussions on the initial model design. This work is funded by the Netherlands Organisation for Scientific Research (NWO).

\section{APPENDIX A: NUMERICAL SOLUTION OF THE SCHRÖDINGER EQUATION IN FRONT OF THE GRATING PATTERN}

We seek a full solution of the (time-independent) Schrödinger equation as a means of obtaining an accurate description of the wave function upon reflection of a (modulated) potential surface. The analysis is based on coupling (known) analytical solutions to Schrödinger's equation for linearly sloped potential fields (region II) to a modulated potential field with unknown solution (region I) as defined in
Fig. 1 of our paper. First, the analytical solution in region II is described. Then, the coupling of this solution to region I is demonstrated and finally the numerical implementation is discussed.

\section{Solutions for a linear sloped potential}

The analytical solution to the Schrödinger equation for a linear sloped potential is obtained by solving the timeindependent Schrödinger equation,

$$
\left[-\frac{\hbar^{2}}{2 m} \nabla^{2}+V(x)\right] \psi(x, y)=\mathcal{E} \psi(x, y) .
$$

The potential-energy function $V(x)$ is dependent on the aperture-mirror separation $d$ and potential difference $\Delta U$. In the absence of a pattern, i.e., a flat mirror, and assuming a grounded aperture, the potential is described as $U(x)=\bar{E} x+$ $U_{p}$ with $\bar{E}$ the linear field strength $(\mathrm{V} / \mathrm{m})$ and $V(x)=e U(x)$. Here, $e=-1.6 \times 10^{-19} \mathrm{C}$ represents the electron charge. The general solution to the resulting differential equation is given by Airy functions of first and second kind [11]. As the solution has to vanish for $x>0$, the Airy function of first kind (from now on referred to as "the Airy function") is the only valid solution of the Schrödinger equation here.

For two spatial dimensions, the solution is given by the product of the Airy function in the direction of the linear sloped potential and plane waves in the direction perpendicular to the ramp; thus

$$
\psi(x, y)=X(x) Y(y)=\operatorname{Ai}\left(u_{n}(x)\right) \exp \left(i k_{y, n} y\right) .
$$

The Airy function $\operatorname{Ai}\left(u_{n}(x)\right)$ is a standing wave type, which is described by

$$
\operatorname{Ai}\left(u_{n}\right) \equiv \frac{1}{\pi} \int_{0}^{\infty} \cos \left(\frac{s^{3}}{3}+s u_{n}\right) d s .
$$

The term $u_{n}(x)$ is a scaling factor that depends on the parameters of the pattern. The derivation of this term is provided inside Appendix B, and given here by

$$
u_{n}(x)=\alpha\left[x-\frac{\mathcal{E}-V_{p}}{E}+\frac{\hbar^{2} k_{y, n}^{2}}{2 m E}\right] .
$$

This expression is obtained by solving the Schrödinger equation analytically in two spatial dimensions by the method of separation of variables. In this equation, $\alpha \equiv\left(2 m E / \hbar^{2}\right)^{1 / 3}$ is a constant that depends only on the linear field strength $E$ in region II. The second term in brackets is recognized as the "classical turning point." This is the coordinate plane where classically the electron would be reflected. However, quantum mechanically there is a finite probability for the electron to penetrate this barrier. Moreover, the electron spends most time just in front of the classical turning point [12]. This is also visible in Fig. 5(a), where the square of the Airy function is plotted as a function of position in front of the mirror plane. The last term in brackets is considered a correction due to the transverse component $k_{y, n}$ of the wave and $m=$ $9.1 \times 10^{-31} \mathrm{~kg}$ is the electron rest mass. 


\section{Coupling the linear and modulated potential region}

The numerical solution of the Schrödinger equation in region $I$ is obtained by a finite element method and described as a boundary value problem. By virtue of the periodicity of the pattern in transverse direction, we only require a single pitch and may apply periodic boundary conditions to both sides. A zero-boundary condition is applied sufficiently far below the classical turning point resulting in full reflection.

The boundary values at each point of the interface of regions I and II are described by the incident and reflected part of the wave function at this boundary,

$$
\psi_{b}=\psi_{\text {incident }}+\psi_{\text {reflected }} \text {. }
$$

For this, the standing-wave-type Airy function must be split in countertraveling components. The asymptotic approximation of the Airy function, valid for $u_{n}>\pi$, allows for this [13], since

$$
\operatorname{Ai}\left(-u_{n}\right) \approx \frac{\sin (\gamma)}{\sqrt{\pi} u_{n}^{1 / 4}}=\underbrace{\frac{\exp (i \gamma)}{2 i \sqrt{\pi} u_{n}^{1 / 4}}}_{\mathrm{Ai}^{+}\left(u_{n}\right)}-\underbrace{\frac{\exp (-i \gamma)}{2 i \sqrt{\pi} u_{n}^{1 / 4}}}_{\operatorname{Ai}^{-}\left(u_{n}\right)} .
$$

The result after the equality sign follows from applying Euler's identity, using $\gamma \equiv \frac{2}{3} u_{n}^{3 / 2}+\pi / 4$ as shorthand notation. This allows us to describe the wave function at the interface in general by

$$
\begin{aligned}
\psi_{b}\left(x_{b}, y\right)= & \sum_{n} A_{n} \mathrm{Ai}^{-}\left(u_{n}\left(x_{b}\right)\right) \exp \left(i k_{y, n} y\right) \\
& +\sum_{n} B_{n} \mathrm{Ai}^{+}\left(u_{n}\left(x_{b}\right)\right) \exp \left(i k_{y, n} y\right) .
\end{aligned}
$$

The complex coefficients $A_{n}$ and $B_{n}$ provide the probability amplitudes for various wave numbers $k_{n}$.

\section{Numerical implementation}

The solution at the interface [Eq. (A5)] is a linear combination of the wave(s) incident towards and reflected by the patterned mirror. The allowed values for the transverse wave component $k_{y, n}$ are determined by the pitch of the mirror pattern and must yield wave numbers that exactly fit an integer amount of times within the pitch. As a result, the interface between regions I and II must consist of $N_{y}$ (necessarily odd) number of points, resulting in allowed values of $k_{y, n}= \pm 2 \pi n$, with $n=0,1, \ldots,\left(N_{y}-1\right) / 2$, in order to prevent numerical aliasing.

The solution to the Schrödinger equation within the proximity of the pattern can now be obtained by a finite difference scheme. Grid points are labeled $\psi_{i, j}$, with $i=1 \ldots N_{x}$ (rows), $j=1 \ldots N_{y}$ (columns), and the grid size is given by $h_{x}$ and $h_{y}$. The full domain consists of $N_{x} \times N_{y}$ grid points. Elements

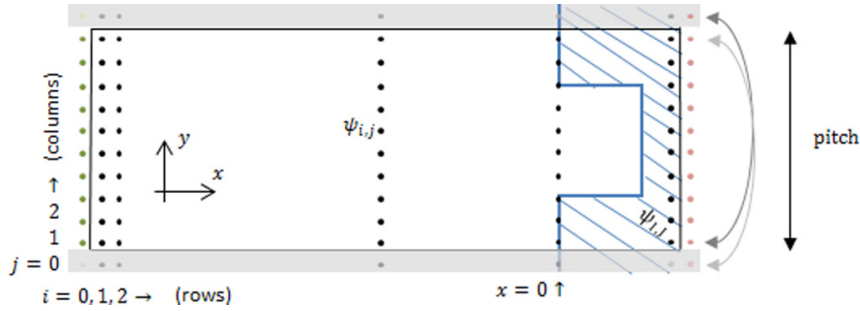

FIG. 6. Schematic representation of the grid, showing the single pitch, boundary conditions, and the labeling of index points.

$\psi_{1, j}$ represent the first row of grid points of region I, directly below the interface. The grid is shown in Fig. 6 .

The Laplacian operator in the kinetic part of the Hamiltonian $\mathbf{H}$ is described by

$$
\begin{aligned}
\nabla_{i, j}^{2} \psi_{i, j}= & \frac{\psi_{i+1, j}+\psi_{i-1, j}-2 \psi_{i, j}}{h_{x}^{2}} \\
& +\frac{\psi_{i, j+1}+\psi_{i, j-1}-2 \psi_{i, j}}{h_{y}^{2}}
\end{aligned}
$$

This introduces a nearest-neighbor dependence. When the grid points $\psi_{i, j}$ are represented by a column vector $\bar{\psi}=\left(\psi_{1,1}, \ldots, \psi_{1, N_{y}}, \psi_{2,1}, \ldots, \ldots, \psi_{N_{x}, N_{y}}\right)^{T}$ the Hamiltonian can be described as a sparse square matrix.

The above definition for the Laplace operator introduces the interface grid points $\psi_{b}$ into the system of equations, since the interface coincides with $\psi_{0, j}$. In turn, this introduces the unknown coefficients $A_{n}$ and $B_{n}$ into the set of equations and the resulting system becomes underdetermined, since we now have $N_{x} \times N_{y}$ equations and (when we choose the incident wave form coefficients $\left.A_{n}\right) N_{x} \times\left(N_{y}+1\right)$ unknowns, due to the unknown coefficients $B_{1}, \ldots, B_{N_{y}}$.

To overcome this problem, one more step is required. By describing the first row of grid points inside the numerical domain (grid elements $\psi_{1, j}$ ) in terms of a Taylor expansion of the interface grid points, the first row entries inside the solution column vector $\bar{\psi}$ can be replaced by coefficients $B_{n}$, such that $\bar{\psi} \rightarrow\left(B_{1}, B_{2}, \ldots, B_{N_{y}}, \psi_{2,1}, \ldots, \ldots, \psi_{N_{x}, N_{y}}\right)^{T}$. The Taylor expansion of the interface elements is given by

$$
\psi_{1, j} \approx \psi_{0, j}+\left.\frac{d \psi_{0, j}}{d x}\right|_{\overline{x_{0}}} h_{x},
$$

where the derivative is taken at the halfway point in between $i=0$ and $i=1$. The Schrödinger equation can now be written as a matrix equation by $(\mathbf{H}-\mathbf{I} \mathcal{E}) \bar{\psi}=\vec{X}$ and $\vec{X}$ is determined by the choice of the incident wave condition. If we define $\mathbf{M} \equiv$ $(\mathbf{H}-\mathbf{I} \mathcal{E})$, we will first explicitly state the form of $\mathbf{M} \bar{\psi}=\overrightarrow{\vec{X}}$ in the following set of expressions:

$$
\begin{gathered}
i=1: \quad \sum_{n} B_{n}\left[\psi_{\text {out }}[j, n]\left(-a_{x}-2 a_{y}+V_{1, j}-\mathcal{E}\right)+\psi_{\text {out }}^{\prime}\left(-2 a_{x}-2 a_{y}+V_{1, j}-\mathcal{E}\right)\right. \\
\left.\quad+a_{y}\left(\psi_{\text {out }}[j \pm 1, n]+\psi_{\text {out }}^{\prime}[j \pm 1, n]\right)\right]+\psi_{2, j}\left(a_{x}\right) \\
=-\sum_{n} A_{n}\left[\psi_{\text {in }}[j, n]\left(-a_{x}-2 a_{y}+V_{1, j}-\mathcal{E}\right)+\psi_{\text {in }}^{\prime}[j, n]\left(-2 a_{x}-2 a_{y}+V_{1, j}-\mathcal{E}\right)\right. \\
\left.\quad+a_{y}\left(\psi_{\text {in }}[j \pm 1, n]+\psi_{\text {in }}^{\prime}[j \pm 1, n]\right)\right],
\end{gathered}
$$




$$
\begin{aligned}
& i=2: \sum_{n} B_{n}\left[a_{x}\left(\psi_{\text {out }}[j, n]+\psi_{\text {out }}^{\prime}[j, n]\right)\right]+\psi_{2, j}\left(-2 a_{x}-2 a_{y}+V_{2, j}-\mathcal{E}\right) \\
&+\psi_{3, j}\left(a_{x}\right)+\psi_{j \pm 1,2}\left(a_{y}\right) \\
&=-\sum_{n} A_{n}\left[a_{x}\left(\psi_{\text {in }}[j, n]+\psi_{\text {in }}^{\prime}[j, n]\right)\right], \\
& i \geqslant 3: \quad \psi_{i \pm 1, j}\left(a_{x}\right)+\psi_{i, j}\left(-2 a_{x}-2 a_{y}+V_{i, j}-\mathcal{E}\right)+\psi_{i, j \pm 1}\left(a_{y}\right)=0 .
\end{aligned}
$$

Here, terms $a_{q}$ relate to the step size in the respective direction $(x, y)$. The analytical expression [also see Eq. (A7)] at the interface is given by

$$
\psi_{b} \equiv \psi_{0, j}=\sum_{n}\left[A_{n} \psi_{\text {in }}[j, n]+B_{n} \psi_{\text {out }}[j, n]\right],
$$

that is, for every point on the interface, the contribution of all modes $n$ are summed for the specific position $j$. For an incident plane wave parallel to the optical axis, only $A_{m}=1$ for $n=m$, the mode corresponding to $k_{y, m}=0$, i.e., the mode that has no transverse wave number component. Further, the derivative of $\psi_{\mathrm{in} / \mathrm{out}}[j, n]$ is defined as given by the Taylor expansion evaluated at the $x$ coordinate related to column $j$ and mode $n$. Also, definition of $\psi_{i \pm 1, j} \equiv \psi_{i-1, j}+$ $\psi_{i+1, j}$ and $\psi[j \pm 1, n] \equiv \psi[j+1, n]+\psi[j-1, n]$ allows for shorthand notations.

The right-hand sides for $i=1$ and $i=2$ in Eq. (A10) provide the source terms for $\vec{X}$. From this scheme it is also clear that only the first $2 J$ positions of $\vec{X}$ are filled with nonzero entries.

\section{Obtaining a solution}

Next, the system $\boldsymbol{M} \vec{\psi}=\vec{X}$ must be solved for $\vec{\psi}$. This is done by left multiplication of both sides of the equation by the inverse of matrix $\boldsymbol{M}$,

$$
\boldsymbol{M}^{-1} \boldsymbol{M} \vec{\psi}=\boldsymbol{M}^{-1} \vec{X} \Rightarrow \vec{\psi}=\boldsymbol{M}^{-1} \vec{X} .
$$

By definition of $\vec{\psi}$ the first $J$ elements contain the coefficients of the reflected waves, $B_{1}, B_{2}, \ldots, B_{J}$. Moreover, for transverse waves of the form $Y_{n}(y)=B_{n} \exp \left(i k_{y, n} y\right)$ the coefficients $B_{n}$ are equivalent to the spectrum of outgoing wave vectors with (field free) small angle approximation angle $\theta_{n}=k_{y, n} / k_{x, n}$ with respect to the optical axis. The corresponding values for $k_{x, n}$ are readily obtained via the field-free solution of the Schrödinger equation, $k_{x, n}=2 m \mathcal{E} / \hbar^{2}-k_{y_{n}}^{2}$, and allow one, for instance, to directly obtain the intensity of the various diffraction orders induced by placing a grating mirror.

We use MATLAB to solve the inversion problem, $\bar{\psi}=$ $(\mathbf{H}-\mathbf{I} \mathcal{E})^{-1} \vec{X}$, where the first $N_{y}$ entries of $\bar{\psi}$ directly yield the probability coefficients $B_{n}$ of various reflected wave components.

\section{Stability and convergence}

In region I, the time-independent Schrödinger equation is solved on a uniform grid with step size $h_{x}$ and $h_{y}$ for the longitudinal and transverse direction. The maximum step value of $h_{x}$ is limited by the shortest wavelength that occurs in the numerical domain. This wavelength occurs at the interface (with potential $V_{0, j}$ ), since the electron is decelerated towards the mirror plane. In order to sample $q$ steps within one wavelength $\lambda$ the value of $h_{x}$ is upper bound by

$$
h_{x} \leqslant \frac{\lambda_{e}}{q} \Rightarrow h_{x} \leqslant \frac{1}{q} \frac{\sqrt{2 m\left[\mathcal{E}-V_{0, j}\right]}}{\hbar} .
$$

The step size $h_{y}$, on the other hand, is unconditionally stable as long as the aliasing requirement $\left(n=0,1, \ldots, \frac{N_{y}-1}{2}\right)$ is met. Then, the second spatial derivative is obtained by two central difference operations,

$$
\begin{aligned}
\nabla_{i, j}^{2} \psi_{i, j}= & \frac{\psi_{i+1, j}+\psi_{i-1, j}-2 \psi_{i, j}}{h_{x}^{2}} \\
& +\frac{\psi_{i, j+1}+\psi_{i, j-1}-2 \psi_{i, j}}{h_{y}^{2}} .
\end{aligned}
$$

Here, the wave function on coordinate $(x, y)$ is represented by $\psi_{i, j}$, where $i=1 \ldots I$ (and $I \equiv N_{x}$ ) labels the row coordinate and $j=1 \ldots J$ (and $J \equiv N_{y}$ ) the column coordinate. Periodic conditions imply that $\psi_{i, J+1}=\psi_{i, 1}$ and $\psi_{i, 0}=\psi_{i, J}$. The zero boundary condition opposite to the interface implies that $\psi_{i, J+1} \equiv 0$.

\section{APPENDIX B: LINEAR SLOPED POTENTIAL SOLUTION FOR TWO-DIMENSIONAL WAVE $\psi(x, y)$}

The solution for a linear sloped potential in the $x$ direction that extends infinitely in the (transverse) $y$ direction is derived here. The potential with slope $E$ (unit $\mathrm{eV} / \mathrm{m}$ ) and mirror pattern potential $V_{p}$ (unit $\mathrm{eV}$ ) is given by

$$
V(x, y)= \begin{cases}0 & \text { for } x \leqslant b \text { with } b<0 \\ E x+V_{p} & \text { for } b<x<0\end{cases}
$$

By using separation of variables $\psi_{I I I}(x, y)=X(x) Y(y)$, the solution in the field-free region (III) follows from the solution of

$$
\frac{1}{X} \frac{d^{2} X}{d x^{2}}+\frac{1}{Y} \frac{d^{2} Y}{d y^{2}}=-\frac{2 m \mathcal{E}}{\hbar^{2}} .
$$

The right-hand side is the separation constant and the corresponding solutions are

$$
\begin{aligned}
& X(x)=A_{\text {in }} \exp \left(i k_{x} x\right)+A_{\text {out }} \exp \left(-i k_{x} x\right), \\
& Y(y)=B_{\text {in }} \exp \left(i k_{y} y\right)+B_{\text {out }} \exp \left(-i k_{y} y\right),
\end{aligned}
$$


with $k_{x}^{2}+k_{y}^{2}=2 m \mathcal{E} / \hbar^{2}$. Next, the solution for the sloped region (II) follows from solving

$$
\begin{aligned}
& \underbrace{-\frac{\hbar^{2}}{2 m} \frac{1}{X} \frac{d^{2} X}{d x^{2}}+V(x)}_{C} \underbrace{-\frac{\hbar^{2}}{2 m} \frac{1}{Y} \frac{d^{2} Y}{d y^{2}}}_{D}=\mathcal{E} \\
& \Rightarrow C+D=\mathcal{E} .
\end{aligned}
$$

Since the potential $V=V(x)$ is only varying in the $x$ direction, it is absorbed in the separation constant $C$, and the solution for $Y(y)$ is equated to a related separation constant $D$ such that $C+D=\mathcal{E}$. The solution for $Y(y)$ is readily obtained,

$$
\begin{aligned}
\frac{d^{2} Y}{d y^{2}} & =-\frac{2 m D}{\hbar^{2}} Y \\
& \Rightarrow Y(y)=D_{1} \exp \left(i k_{y} y\right)+D_{2} \exp \left(-i k_{y} y\right),
\end{aligned}
$$

for given $k_{y}$. Note that $k_{y}$ remains initially a free variable, unless periodic boundary conditions are applied. Then, the values of $k_{y}$ are limited (numerically, for $N_{y}$ is odd) to values $k_{y, n}= \pm 2 \pi n /$ pitch for $n=0,1, \ldots, \frac{N_{y}-1}{2}$, and either $D_{1}=$ 0 or $D_{2}=0$ to avoid double solutions. by

The differential equation describing the $x$ direction is given

$$
\begin{aligned}
\frac{d^{2} X}{d x^{2}} & =-\frac{2 m}{\hbar^{2}}[C-V(x)] X(x) \\
& =-\frac{2 m}{\hbar^{2}}\left[C-E x-V_{p}\right] X(x) \\
& =\frac{2 m E}{\hbar^{2}}\left[x-\frac{C-V_{p}}{E}\right] X(x) .
\end{aligned}
$$

This differential equation can be solved by change of variable. First, define

$$
u=\alpha\left[x-C_{1}\right] \quad \text { with }\left\{\begin{array}{l}
C_{1} \equiv \frac{C-V_{p}}{E}, \\
\alpha \equiv\left(\frac{2 m E}{\hbar^{2}}\right)^{1 / 3} .
\end{array}\right.
$$

Then [14] $d u / d x=\alpha \Rightarrow d^{2} X / d x^{2}=\alpha^{2} d^{2} X / d u^{2}$ and thus the differential equation for $X(x)$ may also be written as

$$
\frac{d^{2} X}{d u^{2}}=u X, \quad \text { with } X=X(u(x)) .
$$

This is a well-known type of differential equation and the solution is given by the Airy functions of first and second kind, respectively $\operatorname{Ai}(u)$ and $\operatorname{Bi}(u)$. Note that the latter grows to infinity for $x>0$ and is no valid solution here. On the other hand, the Airy function of the first kind $\operatorname{Ai}(u(x))$ is a valid solution since it vanishes for $x>0$. The function $\operatorname{Ai}(u)$ is real and defined as

$$
\operatorname{Ai}(u(x))=\frac{1}{\pi} \int_{0}^{\infty} \cos \left(\frac{s^{3}}{3}+s u\right) d s .
$$

The scaling factor $u=u(x)$ is explicitly given by

$$
\begin{aligned}
u_{n}(x) & =\alpha\left[x-C_{1}\right] \\
& =\alpha\left[x-\frac{C}{E}+\frac{V_{p}}{E}\right] \\
& =\alpha\left[x-\frac{2 m \mathcal{E}-\hbar^{2} k_{y, n}^{2}}{2 m E}+\frac{V_{p}}{E}\right] \\
& =\alpha\left[x-\frac{\mathcal{E}-V_{p}}{E}+\frac{\hbar^{2} k_{y, n}^{2}}{2 m E}\right] .
\end{aligned}
$$

In this equation, the term $\left(\mathcal{E}-V_{p}\right) / E$ may be recognized as the "classical turning point" for $1 \mathrm{D}$, that is the position of zero momentum (kinetic energy) of a classical particle. The second term (involving $k_{y, n}^{2}$ ) can be regarded then as a twodimensional energy-conservation correction term. Effectively, this term shifts the point where the Airy function is evaluated in a way that can be regarded as if reflection took place a little earlier (since some of the kinetic energy is in the transverse component now). When desired, the solutions in regions II and III can be connected by equating the normal and first derivative of the wave function at $x=b$ for a selected incident coefficient $A_{\text {in }}$ and $B_{\text {in }}$ for comparison of numerical and analytical results.

\section{APPENDIX C: PHASE ANGLE OF AN ELECTRON REFLECTED FROM A SLOPED POTENTIAL WITH A BUMP}

The presented method is verified against known analytical solutions of the one- and two-dimensional Schrödinger equation. The verification is performed using (i) field free, (ii) constant potential, (iii) linear sloped potential, and (iv) sloped potential with a step edge at the back [12] [Figs. 5(b) and 5(c)].

The following example involves two parts, both in which an incident electron wave is reflected against a linear sloped potential with maximum $V_{\max }>\mathcal{E}$. First, it is shown that the phase angle of the returning wave is obtained, again when this returning wave propagates in a field-free region $(V=0)$ in front of the slope. Next, it is shown that the effect of a perturbation at the top of the slope (near the turning point) can be obtained both analytically and numerically, the latter using the method presented here. A good agreement is obtained for this method.

For a plane incident wave (in field-free region) that is reflected by a linear sloped potential, it can be shown that the analytical solution [for the potential landscape as shown in Fig. 5(b)] to the time-independent Schrödinger equation reads

$$
\psi(x)= \begin{cases}A \exp (i k x)+B \exp (-i k x) & \text { for } V(x)=0, \\ C \operatorname{Ai}(u(x)) & \text { for } V(x)=E x+\mathcal{E} .\end{cases}
$$

The numerical solution is obtained and compared to the analytical solution (for which the value of $B$ and $C$ are uniquely determined for given coefficient $A$ ) and this is shown in Fig. 5(c). Note that the analytical expressions for $B$ and $C$ are obtained 
by equating the expression for the wave function (and the spatial derivative) just to the left and right of the onset for the sloped potential.

In the second part of this example, the validity of the Airy-splitting method is demonstrated [as suggested in Eq. (A6)]. The goal is to obtain the additional phase shift that is due to a potential step (or perturbation) on the top of the linear slope [the orange line in Fig. 5(b)]. An analytical solution for this type of potential (field free, sloped, sloped with additional offset) exists and is given by

$$
\psi(x)= \begin{cases}A \exp (i k x)+B \exp (-i k x) & \text { for } V(x)=0, \\ C \operatorname{Ai}\left(u_{\mathrm{I}}(x)\right) & \text { for } V(x)=E x+\mathcal{E}, \\ D \operatorname{Ai}\left(u_{\mathrm{II}}(x)\right) & \text { for } V(x)=E x+\mathcal{E}(1+\delta) .\end{cases}
$$

Notice that the argument to the Airy function differs for the two parts of the sloped potential. The scaling is given by

$$
u(x)= \begin{cases}\left(\frac{2 m E}{\hbar^{2}}\right)^{1 / 3}(x) & \text { for } u(x)=u_{\mathrm{I}}(x), \\ \left(\frac{2 m E}{\hbar^{2}}\right)^{1 / 3}\left(x+\frac{\delta \mathcal{E}}{E}\right) & \text { for } u(x)=u_{\mathrm{II}}(x) .\end{cases}
$$

When the value of $C_{\text {in }}$ is known (or selected) it is possible to calculate the effect of the offset potential on $C_{\text {out }}$ by starting the calculation just in front of the boundary between the two sloped potentials. The boundary condition $\psi_{0}$ must then discriminate between the incident and reflected component of the Airy function $\operatorname{Ai}\left(u_{\mathrm{I}}\left(x_{0}\right)\right)$, since the Airy function in itself is a real function. This discrimination is made by selecting

$$
\begin{aligned}
\psi_{0} & =C_{\text {in }} \psi_{\text {in }}+C_{\text {out }} \psi_{\text {out }} \\
& =C_{\text {in }} \mathrm{Ai}^{+}\left(u_{0}\right)+C_{\text {out }} \mathrm{Ai}^{-}\left(u_{0}\right),
\end{aligned}
$$

which are left and right traveling waves. Note that the analytical result for $C_{\text {out }}$ is obtained by solving the following system of linear equations:

$$
\left(\begin{array}{cc}
\mathrm{Ai}^{-}\left(u_{\mathrm{I}}\left(x_{s}\right)\right) & -\operatorname{Ai}\left(u_{\mathrm{II}}\left(x_{s}\right)\right) \\
\frac{d}{d x}\left[\mathrm{Ai}^{-}\left(u_{\mathrm{I}}\left(x_{s}\right)\right)\right] & -\frac{d}{d x}\left[\operatorname{Ai}\left(u_{\mathrm{II}}\left(x_{s}\right)\right)\right]
\end{array}\right)\left(\begin{array}{c}
C_{\text {out }} \\
D
\end{array}\right)=\left(\begin{array}{l}
-C_{\text {in }} \mathrm{Ai}^{+}\left(u_{\mathrm{I}}\left(x_{s}\right)\right) \\
-C_{\text {in }} \mathrm{Ai}^{+}\left(u_{\mathrm{I}}\left(x_{s}\right)\right)
\end{array}\right) .
$$

The property of interest now is the phase angle $\phi_{C_{\text {out }}}=\operatorname{Im}\left(C_{\text {out }}\right) / \operatorname{Re}\left(C_{\text {out }}\right)$ and this is shown in Fig. 5(c). Note that the (wrapped) phase angle will not linearly increase with $\delta \mathcal{E}$ when the step size creates a barrier that extends above $V=\mathcal{E}$. This is the case here for $\delta \mathcal{E}=10 \%$ of $\mathcal{E}$.

[1] P. Kruit et al., Ultramicroscopy 164, 31 (2016).

[2] P. W. Hawkes and E. Kasper, Principles of Electron Optics (Academic Press, New York, 1994), Vol. 3.

[3] H. Lichte and G. Möllenstedt, J. Phys. E: Sci. Instrum. 12, 941 (1979).

[4] T. R. Harvey, J. S. Pierce, A. K. Agrawal, P. Ercius, M. Linck, and B. J. McMorran, New J. Phys. 16, 093039 (2014).

[5] Assuming $2 \mathrm{keV}$ beam, $500 \mathrm{~nm}$ pitch, and mirror potential of -2004 V. Numbers are approximations.

[6] P. Machnikowski, Phys. Rev. B 73, 155109 (2006).

[7] J. R. Anglin, J. P. Paz, and W. H. Zurek, Phys. Rev. A 55, 4041 (1997).

[8] P. Sonnentag and F. Hasselbach, Phys. Rev. Lett. 98, 200402 (2007).
[9] B. Barwick et al., J. Appl. Phys. 100, 074322 (2006).

[10] Y. Yang, C.-S. Kim, R. G. Hobbs, P. Kruit, and K. K. Berggren, Phys. Rev. A 98, 043621 (2018).

[11] D. J. Griffiths, Introduction to Quantum Mechanics (Pearson Prentice Hall, Englewood Cliffs, NJ, 2005).

[12] S. M. Kennedy, D. E. Jesson, M. J. Morgan, A. E. Smith, and P. F. Barker, Phys. Rev. A 74, 044701 (2006).

[13] E. T. Copson, Proc. Glasgow Math. Ass. 6, 113 (1963).

[14] It should not be confused that $d^{2} / d u^{2}=(d / d u)^{2}$ in general. This is only coincidental in the case here, since $u$ is only linearly dependent on $x$. This result follows from applying the chain rule for derivation twice. 